\title{
UNICITY THEOREMS FOR MEROMORPHIC FUNCTIONS
}

\author{
Amer H. H. Al-Khaladi*
}

\begin{abstract}
This paper studies the problem of uniqueness of meromorphic functions. In this paper we will improve a result given by $\mathrm{K}$. Tohge.
\end{abstract}

\section{§1. Introduction}

By a "meromorphic function" we will mean a meromorphic function in the complex plane. It is assumed that the reader is familiar with the notations of the Nevanlinna theory that can be found, for instance, in [2] or [4]. Let $f$ and $g$ be two non-constant meromorphic functions and $a$ be a value in the extended complex plane. We say that $f$ and $g$ share a value $a$ IM (ignoring multiplicity), if $f$ and $g$ have the same $a$-points, and also they share the value $a$ CM (counting multiplicity), if $f$ and $g$ have the same $a$-points with the same multiplicity. Let $k$ be a positive integer or $\infty$, we denote by $\bar{E}_{k)}(a, f)$ the set of $a$-points of $f$ with multiplicity $\leq k$ (ignoring multiplicity), by $N_{k}(r, 1 /(f-a))$ the counting function of $a$-points of $f$ with multiplicity $\leq k$ and by $N_{(2}(r, 1 /(f-a))$ the counting function of $a$-points of $f$ with multiplicity $\geq 2$ (See [4]). Finally we say $a$ is a Picard exceptional value of $f$, if $f(z) \neq a$.

In [3] K. Tohge proved the following:

THEOREM 1. Let $f$ and $g$ be non-constant meromorphic functions that share three values $0,1, \infty \mathrm{CM}$ and $f^{\prime}, g^{\prime}$ share $0 \mathrm{CM}$. Then $f$ and $g$ satisfy one of the following:

$$
\begin{aligned}
& \text { (i) } f \equiv g, \\
& \text { (ii) } f g \equiv 1, \\
& \text { (iii) }(f-1)(g-1) \equiv 1, \\
& \text { (iv) } f+g \equiv 1,
\end{aligned}
$$

* Project supported by NSFC and RFDP

1991 Mathematics Subject Classification: 30D35

Received November 16, 1998; revised December 1, 1999. 


$$
\begin{aligned}
& \text { (v) } f \equiv c g, \\
& \text { (vi) } f-1 \equiv c(g-1), \\
& \text { (vii) }[(c-1) f+1][(c-1) g-c] \equiv-c,
\end{aligned}
$$

where $c(\neq 0,1)$ is a constant.

In this paper, we prove the following theorem which is an improvement of Theorem 1 .

THEOREM 2. Let $f$ and $g$ be non-constant meromorphic functions that share two values $0, \infty \mathrm{CM}$ and $f^{\prime}, g^{\prime}$ share the value 0 IM. If $\bar{E}_{k)}(1, f)=\bar{E}_{k)}(1, g)$, where $k$ is a positive integer or $\infty$, then $f$ and $g$ satisfy one of the identities in (1.1).

\section{§2. Some lemmas}

LEMma 1 (See [4]). Let $f$ and $g$ be distinct non-constant entire functions and $a_{1}, a_{2}$ are distinct finite complex numbers. If $a_{1}$ is a Picard exceptional value of $f, g$ and $f, g$ share the value $a_{2} \mathrm{CM}$, then $f=e^{\alpha}+a_{1}$ and $g=\left(a_{1}-a_{2}\right)^{2} e^{-\alpha}+a_{1}$, where $\alpha$ is a non-constant entire function.

LEMMA 2 (See [4]). Let $f$ and $g$ be distinct non-constant meromorphic functions and $a_{1}, a_{2}$ be distinct finite complex numbers. If $a_{1}, a_{2}$ are Picard exceptional values of $f, g$ and $f, g$ share the value $\infty \mathrm{CM}$, then

$$
f=\frac{a_{1} e^{\alpha}-a_{2}}{e^{\alpha}-1}, \quad g=\frac{a_{1} e^{-\alpha}-a_{2}}{e^{-\alpha}-1},
$$

where $\alpha$ is a non-constant entire function.

LEMMA 3 (See [1]). Let $f$ and $g$ be non-constant meromorphic functions that share three values $0,1, \infty \mathrm{CM}$. If $f$ is a Möbius transformation of $g$, then $f$ and $g$ satisfy one of the identities in (1.1).

Proof. Suppose $f \not \equiv g$. Since $f$ is a Möbius transformation of $g$,

$$
g=\frac{a f+b}{c f+d}
$$

where $a, b, c, d$ are finite complex numbers and $a d-b c \neq 0$. There are three cases.

CASE I. If $\infty$ is a Picard exceptional value of $f$, then there are four subcases.

1. If 1 and 0 are Picard exceptional values of $f$, then this case is impossible due to the second fundamental theorem.

2. If 1 and 0 are not Picard exceptional values of $f$, then from (2.1) we get $b=0, c+d=a$ and hence (2.1) becomes 


$$
c f g+d g=(c+d) f .
$$

Since $b=0$ and from $a d-b c \neq 0$ we find $a d \neq 0$ and hence (2.2) becomes

$$
(A-1) f g-A f+g \equiv 0,
$$

where $A=a / d \neq 0$. If $A=1$, then from (2.3) we find $f \equiv g$. This is a contradiction. Therefore $A \neq 1$. Thus from (2.3) we find $[(A-1) f+1]$. $[(A-1) g-A] \equiv-A$, which is (vii).

3. If 0 is a Picard exceptional value of $f$ and 1 is not a Picard exceptional value of $f$, then by Lemma 1 we find $f=e^{\alpha}$ and $g=e^{-\alpha}$. From this we find $f g \equiv 1$, which is (ii).

4. If 1 is a Picard exceptional value of $f$ and 0 is not a Picard exceptional value of $f$, then by Lemma 1 we find $f=e^{\alpha}+1$ and $g=e^{-\alpha}+1$. From this we find $(f-1)(g-1) \equiv 1$, which is (iii).

CASE II. If 0 is a Picard exceptional value of $f$, then there are two subcases.

1. If $\infty$ and 1 are not Picard exceptional values of $f$, then from (2.1) we find $c=0$ and $a+b=d$. Again by (2.1) we get $f-1=A(g-1)$ where $A(\neq 0,1)$ is a constant, which is (vi).

2. If 1 is a Picard exceptional value of $f$ and $\infty$ is not a Picard exceptional value of $f$, then by Lemma 2 we find $f=-1 /\left(e^{\alpha}-1\right)$ and $g=-1 /\left(e^{-\alpha}-1\right)$. From this we find $f+g \equiv 1$, which is (iv).

CASE III. If 1 is a Picard exceptional value of $f$, then there is only one subcase: If $0, \infty$ are not Picard exceptional values of $f$, then by $(2.1)$ we find $b=c=0$ and hence (2.1) becomes $f \equiv A g$, which is (v).

\section{§3. Proof of Theorem 2}

From the conditions of Theorem 2 we find

$$
f=e^{\alpha} g
$$

where $\alpha$ is an entire function. If $e^{\alpha} \equiv c$, where $c$ is a nonzero constant, then from this and (3.1) we deduce (v). We now suppose $e^{\alpha}$ is non-constant and hence $\alpha^{\prime} \not \equiv 0$. Again from (3.1) we have

$$
f^{\prime}=g^{\prime} e^{\alpha}+g \alpha^{\prime} e^{\alpha}
$$

Let $z_{0}$ be a zero for $f^{\prime}$ of order $p \geq 1$, then the Taylor expansion of $f^{\prime}$ about $z_{0}$ is

$$
f^{\prime}(z)=a_{p}\left(z-z_{0}\right)^{p}+\cdots, \quad a_{p} \neq 0 .
$$

Since $f^{\prime}$ and $g^{\prime}$ share the value 0 IM, therefore

$$
g^{\prime}(z)=b_{q}\left(z-z_{0}\right)^{q}+\cdots, \quad b_{q} \neq 0 .
$$


Without loss of generality, we can assume that $p \leq q$. From (3.2), (3.3) and (3.4) we find

$$
g(z) \alpha^{\prime}(z)=\left(z-z_{0}\right)^{p}\left[a_{p} e^{-\alpha}-b_{q}\left(z-z_{0}\right)^{q-p}+O\left(z-z_{0}\right)\right] .
$$

From this we find either $\alpha^{\prime}\left(z_{0}\right)=0$ or $g\left(z_{0}\right)=0$. If $g\left(z_{0}\right)=0$ then $p=q$. Thus we find

$$
\bar{N}\left(r, \frac{1}{f^{\prime}}\right)-N_{E}^{\prime}(r, 0) \leq N\left(r, \frac{1}{\alpha^{\prime}}\right) \leq T\left(r, \alpha^{\prime}\right)+O(1) \leq S(r, f)+S(r, g),
$$

where $N_{E}^{\prime}(r, 0)$ denotes the counting function of zeros of $f^{\prime}$ and $g^{\prime}$ with same multiplicity, each zero being counted only once. Similarly with respect to $g^{\prime}$ we find

$$
\bar{N}\left(r, \frac{1}{g^{\prime}}\right)-N_{E}^{\prime}(r, 0)=S(r, f)+S(r, g) .
$$

Let $z_{1}$ be a zero for $f-1$ of order $p \geq 2$. Then $z_{1}$ is also a zero for $f^{\prime}$ and hence for $g^{\prime}$. From (3.2) we find $\alpha^{\prime}\left(z_{1}\right)=0$. From this we find

$$
\bar{N}_{(2}\left(r, \frac{1}{f-1}\right) \leq N\left(r, \frac{1}{\alpha^{\prime}}\right) \leq T\left(r, \alpha^{\prime}\right)+O(1) \leq S(r, f)+S(r, g) .
$$

Similarly with respect to $g$ we find

$$
\bar{N}_{(2}\left(r, \frac{1}{g-1}\right)=S(r, f)+S(r, g) \text {. }
$$

We denote by $N_{1)}(r, 1)$ the counting function of common simple 1-points of $f$ and $g$. Noting $\bar{E}_{k)}(1, f)=\bar{E}_{k)}(1, g)$, from (3.7) and (3.8), we have

$$
\bar{N}\left(r, \frac{1}{f-1}\right)-N_{1)}(r, 1)=S(r, f)+S(r, g)
$$

and

$$
\bar{N}\left(r, \frac{1}{g-1}\right)-N_{1)}(r, 1)=S(r, f)+S(r, g)
$$

Set

$$
\Delta_{1}=\frac{f^{\prime \prime}}{f^{\prime}}-\frac{g^{\prime \prime}}{g^{\prime}} .
$$

From the fundamental estimate of logarithmic derivative it follows that

$$
m\left(r, \Delta_{1}\right)=S(r, f)+S(r, g) .
$$

From (3.11) we find 


$$
N\left(r, \Delta_{1}\right) \leq \bar{N}\left(r, \frac{1}{f^{\prime}}\right)-N_{E}^{\prime}(r, 0)+\bar{N}\left(r, \frac{1}{g^{\prime}}\right)-N_{E}^{\prime}(r, 0) .
$$

From (3.5), (3.6), (3.12) and (3.13) we find

$$
T\left(r, \Delta_{1}\right)=S(r, f)+S(r, g) \text {. }
$$

Let $z_{\infty}$ be a simple pole of $f$, then from (3.11) we find

$$
\Delta_{1}\left(z_{\infty}\right)=0 .
$$

If $\Delta_{1} \equiv 0$, then from (3.11) we find

$$
f=a g+b,
$$

where $a(\neq 0), b$ are constants. If $b=0$, then from (3.16) we deduce (v). We now suppose $b \neq 0$. Since $\bar{E}_{k)}(1, f)=\bar{E}_{k)}(1, g)$, therefore, if $\bar{N}_{k)}(r, 1 /(f-1)) \not \equiv$ 0 , then from (3.16) we find $a+b=1$. From this and (3.16) we deduce (vi). We now suppose

$$
\bar{N}_{k)}\left(r, \frac{1}{f-1}\right)=\bar{N}_{k)}\left(r, \frac{1}{g-1}\right) \equiv 0 .
$$

From (3.1) and (3.16) we find

$$
f-1=\frac{(b-1) e^{\alpha}+a}{e^{\alpha}-a} \text { and } g-1=\frac{a+b-e^{\alpha}}{e^{\alpha}-a} .
$$

From this and (3.17) we find $b=1$ and $a=-1$. From this and (3.18) we deduce (iv). We now suppose $\Delta_{1} \not \equiv 0$. From (3.14) and (3.15) we find

$$
N_{1)}(r, f) \leq N\left(r, \frac{1}{\Delta_{1}}\right) \leq T\left(r, \Delta_{1}\right)+O(1) \leq S(r, f)+S(r, g) \text {. }
$$

Set

$$
\Delta_{2}=\frac{f^{\prime}}{f-1}-\frac{f^{\prime}}{f}-\frac{g^{\prime}}{g-1}+\frac{g^{\prime}}{g} .
$$

From the fundamental estimate of logarithmic derivative it follows that

$$
m\left(r, \Delta_{2}\right)=S(r, f)+S(r, g) .
$$

From (3.9), (3.10), (3.20) and (3.21) we find

$$
T\left(r, \Delta_{2}\right)=S(r, f)+S(r, g) .
$$

Let $z_{\infty}^{\prime}$ be a pole for $f$ of order $p \geq 2$. Then from (3.20) we find

$$
\Delta_{2}\left(z_{\infty}^{\prime}\right)=0 .
$$

If $\Delta_{2} \equiv 0$, then from $(3.20)$ we find 


$$
\frac{f-1}{f}=c \frac{g-1}{g},
$$

where $c$ is a nonzero constant. From this it is easy to see $f$ and $g$ share the value 1 CM. And hence from (3.24) and Lemma 3 we find $f$ and $g$ satisfy one of the identities in (1.1). We now suppose $\Delta_{2} \not \equiv 0$. From (3.22) and (3.23) we find

$$
N_{(2}(r, f) \leq 2 N\left(r, \frac{1}{\Delta_{2}}\right) \leq 2 T\left(r, \Delta_{2}\right)+O(1) \leq S(r, f)+S(r, g) .
$$

From (3.19) and (3.25) we find

$$
N(r, f)=S(r, f)+S(r, g) \text {. }
$$

Set

$$
\Delta_{3}=\frac{f^{\prime \prime}}{f^{\prime}}-2 \frac{f^{\prime}}{f}-\frac{g^{\prime \prime}}{g^{\prime}}+2 \frac{g^{\prime}}{g} .
$$

Similar to the above, from (3.5), (3.6) and (3.27) it is easy to see that

$$
T\left(r, \Delta_{3}\right)=S(r, f)+S(r, g) .
$$

Let $z_{0}$ be a simple zero of $f$. Then from (3.27) we find

$$
\Delta_{3}\left(z_{0}\right)=0 \text {. }
$$

If $\Delta_{3} \equiv 0$, then from (3.27) we easily arrive at that $f$ and $g$ satisfy one of identities in (1.1). We now suppose $\Delta_{3} \not \equiv 0$. Then from (3.28) and (3.29) we find

$$
N_{1)}\left(r, \frac{1}{f}\right)=S(r, f)+S(r, g) \text {. }
$$

Set

$$
\Delta_{4}=\frac{f^{\prime}}{f-1}-\frac{g^{\prime}}{g-1}
$$

Again by a similar way as the above, we find from (3.9), (3.10) and (3.31) that

$$
T\left(r, \Delta_{4}\right)=S(r, f)+S(r, g) \text {. }
$$

Let $z_{0}^{\prime}$ be a zero for $f$ of order $p \geq 2$. Then from (3.31) we find

$$
\Delta_{4}\left(z_{0}^{\prime}\right)=0 \text {. }
$$

If $\Delta_{4} \equiv 0$, then from (3.31) we easily arrive at that $f$ and $g$ satisfy one of the identities in (1.1). We now suppose $\Delta_{4} \not \equiv 0$. Then from (3.32) and (3.33) we find

$$
N_{(2}\left(r, \frac{1}{f}\right)=S(r, f)+S(r, g) .
$$

And hence from (3.30) and (3.34) we find 


$$
N\left(r, \frac{1}{f}\right)=S(r, f)+S(r, g) \text {. }
$$

Set

$$
\Delta_{5}=\frac{f^{\prime \prime}}{f^{\prime}}-2 \frac{f^{\prime}}{f-1}-\frac{g^{\prime \prime}}{g^{\prime}}+2 \frac{g^{\prime}}{g-1}
$$

Similar to the above we find from (3.5), (3.6), (3.9), (3.10) and (3.26) that

$$
T\left(r, \Delta_{5}\right)=S(r, f)+S(r, g) .
$$

Let $z_{1}$ be a common simple 1-point of $f$ and $g$. Then from (3.36) we find

$$
\Delta_{5}\left(z_{1}\right)=0 .
$$

If $\Delta_{5} \equiv 0$, then from (3.36) we easily arrive at that $f$ and $g$ satisfy one of the identities in (1.1). We now suppose $\Delta_{5} \not \equiv 0$. Then from (3.37) and (3.38), we find

$$
\begin{aligned}
N_{1)}(r, 1) \leq N\left(r, \frac{1}{\Delta_{5}}\right) & \leq T\left(r, \Delta_{5}\right)+O(1) \\
& \leq S(r, f)+S(r, g) .
\end{aligned}
$$

From this and (3.9), we have

$$
\bar{N}\left(r, \frac{1}{f-1}\right)=S(r, f)+S(r, g) .
$$

Similarly, we get

$$
\bar{N}\left(r, \frac{1}{g-1}\right)=S(r, f)+S(r, g) .
$$

Thus from (3.26), (3.35), (3.39), (3.40) and the second fundamental theorem for $f$ and $g$ we find

$$
\begin{aligned}
T(r, f)+T(r, g) \leq & 2 \bar{N}\left(r, \frac{1}{f}\right)+\bar{N}\left(r, \frac{1}{f-1}\right)+\bar{N}\left(r, \frac{1}{g-1}\right) \\
& +2 \bar{N}(r, f)+S(r, f)+S(r, g) \\
\leq & S(r, f)+S(r, g),
\end{aligned}
$$

this is impossible. And so the proof of Theorem 2 is finished.

Acknowledgement. I am very grateful to professor Hong-Xun Yi for helpful direction and the referee for valuable comments. 


\section{REFERENCES}

[1] A. H. H. Al-Khaladi, Meromorphic functions that share three values with one share value for their derivatives, to appear.

[2] W K. Hayman, Meromorphic Functions, Clarendon Press, Oxford, 1964.

[3] K. Tohge, Meromorphic functions covering certain finite sets at the same points, Kodai Math. J., 11 (1988), 249-279.

[4] H. X. Yi AND C. C. YANG, Uniqueness Theory of Meromorphic Functions, Science Press, Beijing, 1995.

Department of Mathematics

SHANDONG UNIVERSITY

JinAN, ShandONG 250100

People's Republic of China 